\title{
Rare case report of Pseudopseudohypoparathyroidism (PPHP) with uretric calculi
}

\begin{abstract}
Hypoparathyroidism (HP) is characterised by low level of parathyroid hormone (PTH), low calcium and high phosphate with normal phenotype. Pseudohypoparathyroidism (PHP) is characterised by resistance to PTH and therefore PTH level are high along with low calcium and high phosphate level and sometime this is associated with Albright hereditary osteodystrophy (AHO) phenotype, especially in type 1A of PHP. Pseudopseudohypoparathyroidism (PPHP) is an inherited disorder, is biochemically normal but phenotypically similar to pseudohypoparathyroidism type $1 \mathrm{a}$, called Albright hereditary osteodystrophy (AHO) phenotype. AHO phenotype was first characterized in 1952 by Fuller Albright as "pseudo-pseudohypoparathyroidism". Renal stone is a rare phenomenon in PPHP and there is only one case report in our knowledge so far in literature. We have seen a 27 year old unmarried male, presented with low back pain associated with restriction of neck movement and bilateral elbow movement from last 8 years with progressive course. History of renal colic was also there. There was positive family history of similar illness in younger brother. On examination there was restriction of spinal mobility, Short 4th and 5th fingers, Knuckle knuckle dimple dimple sign. On radiological evaluation generalized heterotopic calcification involving cervical spine, hip and elbow was present with renal stone. Short 4th and 5th metacarpals and short metatarsals with Exostosis around 1st metacarpal was seen.Biochemical investigations showed normal levels of serum calcium, phosphate, parathyroid hormone, thyroid stimulating hormone, vitamin D levels.Occurrence of renal stone can be there in PHP but very rare in PPHP. Diagnosis of psudopsudohypoparathrodism is made on the basis of early age of onset with progressive course, restriction of spinal mobility, short 4th and 5th fingers ,knuckle knuckle dimple dimple sign, history of uretric colic and renal stone .Generalized heterotopic calcification on radiological investigations without any evidence of hormone resistance with normal serum calcium, phosphate along with positive family history. Patients with Pseudopseudohypoparathyroidism usually do not need specific treatment or further follow-up, but if symptomatic, treatment of related complication can be considered depending upon situations.
\end{abstract}

Keywords: selective transfer, superficial layer, structural analysis, intensity x-rays, width of diffraction lines, crystalline network constant
Volume 10 Issue 2 - 2018

\author{
Rakesh Kumar Jagdish,' Shailly, ${ }^{2}$ MK \\ Bhatnagar $^{3}$ \\ 'Consultant Rheumatologist, Kailash and Max Hospital, Noida. \\ Ex. Senior Resident Rheumatology, AlIMS, India \\ ${ }^{2}$ Resident TB and Chest Hospital, Government Medical College \\ Patiala, India \\ ${ }^{3}$ Professor of Medicine Santosh Medical College and Hospital \\ Ghaziabad, Ex Director Professor Lady Hardinge Medical \\ College, India
}

\section{Correspondence: Rakesh Kumar Jagdish, Consultant} Rheumatologist, Kailash and Max Hospital, Noida, Ex. Senior Resident Rheumatology, AlIMS, Delhi, India, Email dr.rkj.kapil@gmail.com

Received: July II, 2017 | Published: April 12, 2018

\section{Case report}

A 27 year old unmarried male, resident of Agartala, India, a clerk by occupation non smoker, non alcoholic, presented with low back pain associated with restriction of neck movement and bilateral elbow from last 8 years. Patient was asymptomatic till 18 years of age, then he develops gradually progressive mechanical type of low back pain, associated with pain and restriction of movement of both elbow with restricted movement of spine, involving cervical, thoracic and lumbar spine. There was no history suggestive of spondyloarthritis $(\mathrm{SpA})$ features like enthesitis, dactylitis, uveitis, and psoriasis, loose stool, bleeding per rectum, alternating buttock pain, night pains, early morning pain and stiffness. He never had paresthesias, muscular twitching, convulsions or tetany. Milestone development and onset of puberty were normal. There was no problem with vision. No problem in teeth, gastrointestinal disturbance, hypertension, Diabetes, kidney diseases, previous hospitalization and blood transfusion. There was positive family history of similar illness in younger brother. He had acute uretric colic 8 to 9 month before which was diagnosed as left sided lower uretric calculi and treated conservatively

On examination he has normal built and normal vitals with normal orientation and mentation. Skin, hair and nails normal .Sclera and fundus examination was normal. Thyroid and JVP were normal. There was no pallor, icterus, cyanosis, clubbing, lymphadenopathy and oedema. Chest, cardiovascular and abdominal examination was within the normal limits. Musculoskeletal examination: Restriction of spinal mobility (cervical and lumbosacral) was there with flexion deformity both elbows (right $>$ left) .Other joints were normal.

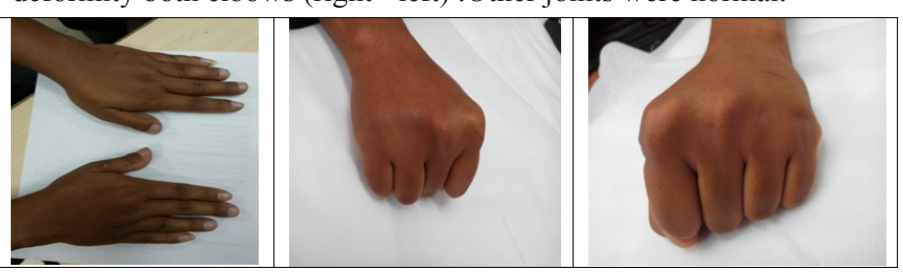

Figure I-3 Short $4^{\text {th }}$ and $5^{\text {th }}$ fingers than normal person. Knuckle knuckle dimple dimple sign. 


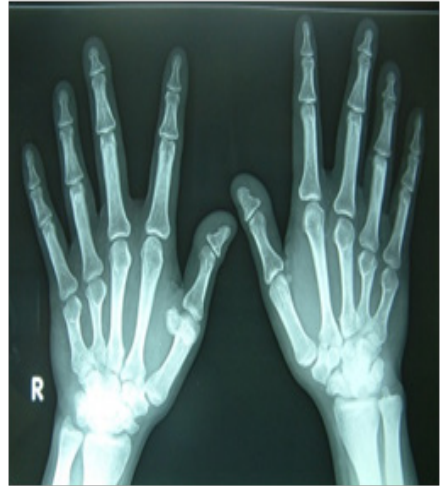

Figure $4 \times$ ray hands: Short $4^{\text {th }}$ and $5^{\text {th }}$ metacarpals, large exostosis at distal end of Ist right metacarpals, both wrist normal.

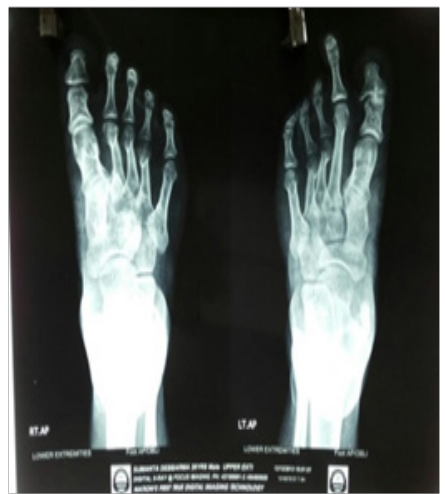

Figure $5 \times$ ray feet: Right $4^{\text {th }}$ and $5^{\text {th }}$ metatarsal short and left $2^{\text {nd }}, 3^{\text {rd }}$, and $4^{\text {th }}$ metatarsal short.

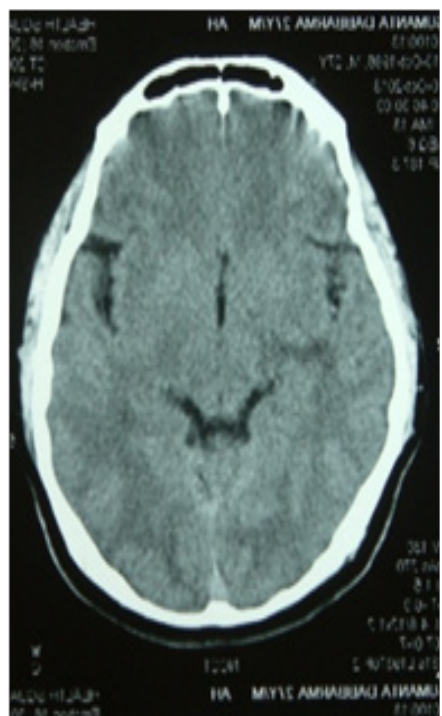

Figure 6 Brain imaging-normal, no basal ganglia calcification.

Routine lab tests e.g. complete blood count, ESR,CRP, Renal function test including electrolytes, liver function tests including enzymes, Thyroid function tests ,PTH, Vitamin D3 level urine examination, sugar level all were in normal range. Autoimmune work up e.g. Rheumatoid factor, anti-CCP, Anti nuclear antibody (ANA), HLA B27 all were negative. Ophthalmological examination, 2D Echocardiography, chest X ray, MRI brain all was normal. Other radiological studies showed heterotopic calcification and short metacarpals and metatarsals, as shown in images. Ellsworth-Howard test: was not done ,as clinically and by blood testing no evidence of hormone resistance present and foremost importance it was not available in our hospital and patient was not affording.Genetic tests for mutation of the GNAS gene was not done, as facilities was not available. Diagnosis of psudopsudohypoparathrodism was made on the basis of above clinical features and investigations.Other features of AHO phenotype like short stature, round face, obesity and mental retardation are not necessary to make diagnosis of it (Figures 1-9). ${ }^{1,2}$

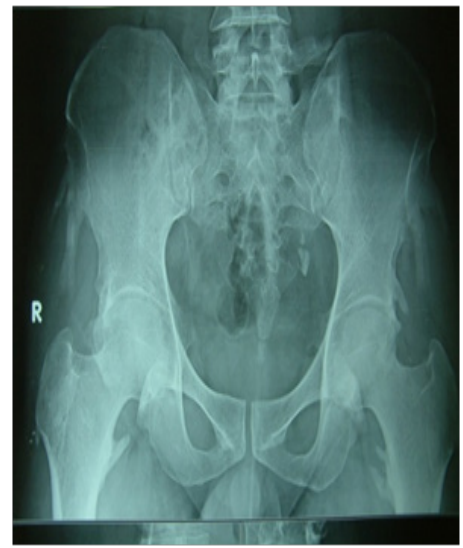

Figure $7 \times$ ray pelvis: multiple soft tissue calcification- ligamental calcifications at different ligamental insertion sites. Left sided radio opacities (uretric calculi),No evidence of sacroilitis.

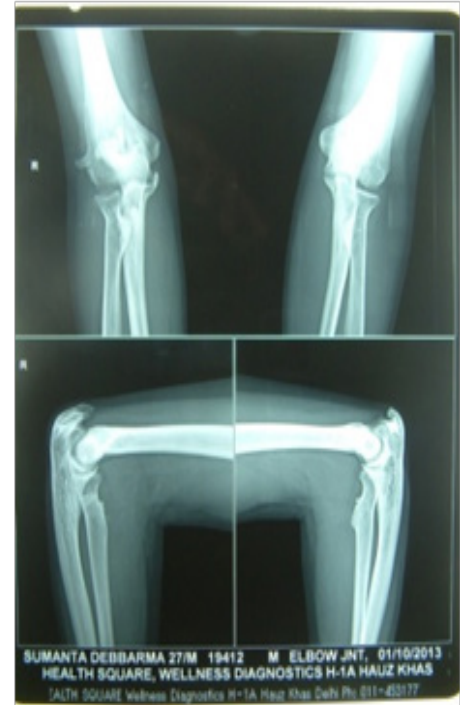

Figure $8 \times$ ray both elbow: ligament/tendon ossification (calcification) at insertion sites 


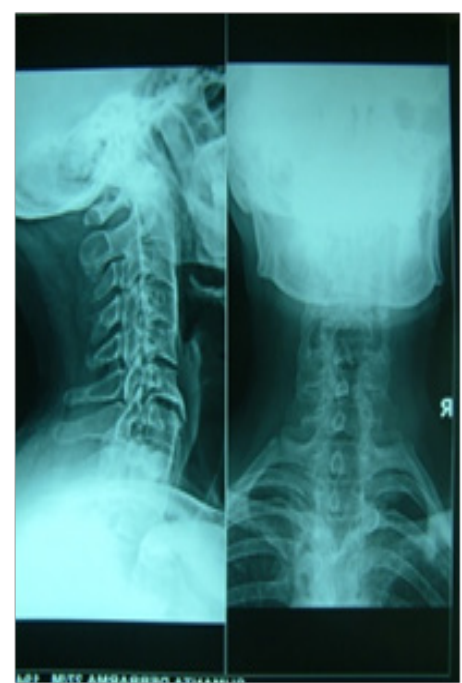

Figure 9 X-ray cervical spine: anterior and posterior longitudinal ligament calcification upto $\mathrm{c} 5$.

C6- osteophytes formation

\section{Discussion}

Image 10 is showing the differentiation between hypoparathyroidism (HP), pseudohypoparathyroidism (PHP) and Pseudopseudohypoparathyroidism (PPHP). Genetic problem in PHP/ PPHP is a copying error (often called mutation) in GNAS1 gene. People who inherit the mutation from their mother develop PHP whereas those who inherit the mutation from their father develop $\mathrm{PPHP}^{3}$ or when not inherited then likely a new mutation has been formed when the baby was conceived. Autosomal dominant pattern with genetic imprinting phenomenon, which explains the variations in phenotype depending upon the maternal or paternal mutation's origin (Table 1). ${ }^{4}$

\section{Albright Hereditary Osteodystrophy (AHO)}

PHP is historically the first hormone resistance syndrome, and it was described for the first time in 1942 by Albright et al. ${ }^{5}$ AHO initially described together with PHP in 1942, which encompasses heterogeneous clinical findings such as brachydactyly, rounded face, short stature, central obesity, subcutaneous ossifications, and variable degrees of mental retardation. ${ }^{6,7}$, Brachydactyly, , classically described as a shortening of III, IV, and V metacarpals and I distal phalanx, is the typical and, together with heterotopic ossifications, the most specific feature of the AHO phenotype. ${ }^{2} \mathrm{AHO}$ prevalence is approximately 0.79 per 100,000 (according to orphanet report series, November 2008).Mental retardation - Variable and inconsistent presentation, adult $(27 \%)$ and the paediatric population $(64 \%)^{8,9}$ Short stature, obesity, round face and delayed onset of puberty are mostly associated with AHO phenotype associated with PHP 1a, not regularly with PPHP, likely due to multiple hormone resistance in PHP 1a and defective signaling of Gs in other cells of body for both of these As for obesity, despite being classically included among AHO signs, it has recently been shown to be associated with PHP-Ia more than AHO it self. ${ }^{10}$ Although exact cause and mechanism is not still clear why so much variability in phenotypical appearance. as, patients showing the physical features of AHO without any evidence of PTH resistance were also described by Albright et al. ${ }^{11}$ The AHO phenotype results from deficient signalling in cells in which the Gs $\alpha$ gene is haploinsufficient but does not suffer imprinting (both alleles are expressed). ${ }^{12}$ The diminution of signalling Gs $\alpha$ appears to promote osteoblastic differentiation, which may explain ectopic calcifications and premature closing of growth plates. ${ }^{13}$

\section{Table I HP/PHP/PPHP}

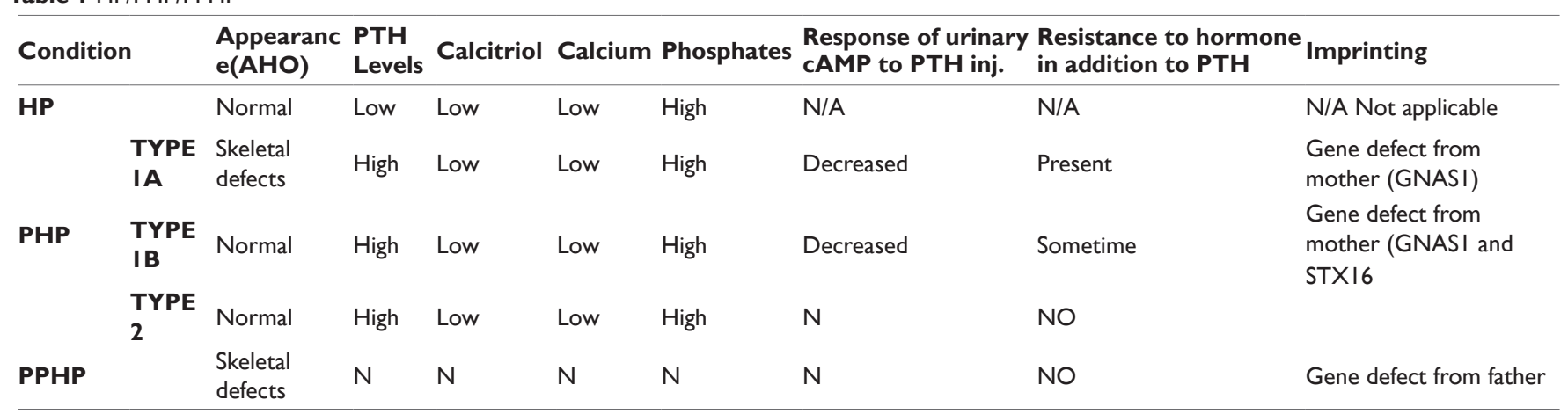

Since the genetic defect in type 2 is further down the signalling pathway than in type I, there is a normal response to PTH stimulation despite the inherent abnormality in calcium regulation.

\section{Other differential diagnosis which were considered (Table 2)}

\section{Possible causes for renal stone disease in present case:}

Unlike the automatic actions of parathyroid hormone, the vitamin $\mathrm{D}$ analogue is not able to either regulate or precisely maintain the level of calcium in the blood nor can it influence the kidneys to reabsorb calcium and 'recycle' it back into the blood stream. This results in larger than normal amounts of calcium passing through the renal system and being excreted in urine and increased risk of renal problems or stones. In Nov 29, 1954, MARCEL ROCHE publish a paper on Pseudopseudohypoparathyroidism (PPHP) of a 40 year old male patient with history of uretric colic but not able to demonstrate stone in x ray as it might had passed away through urine, as pain subsided after patient admitted in hospital. This is the only partial report of uretric stone with PPHP available to me on search. ${ }^{14}$ 
Table 2 Differential diagnosis

\begin{tabular}{ll}
\hline Causes of heterotopic calcification & Cause of short metacarpals \\
\hline $\begin{array}{ll}\text { Post traumatic (fracture, contusion, myositis ossificans } \\
\text { traumatica, post operative after hip replacement surgery). }\end{array}$ & AHO (Albright hereditary osteodystrophy). \\
Neurogenic: spinal trauma, head injury. & Post traumatic. \\
CKD and associated tertiary hyperparathyroidism. & Post infective (TB, dactylitis, osteomyelitis). \\
Fibro dysplasia ossificans progressive. & Turner syndrome/Down syndrome. \\
Progressive osseous hetroplasia. & Sickle cell disease with secondary infracts. \\
AHO (Albright hereditary osteodystrophy). & Hereditary exostosis syndrome. \\
Hypervitaminosis A. & Homocystinuria. \\
Hypervitaminosis D. & Langer giedion syndrome. \\
Fluorosis. & Isolated shorting of $5^{\text {th }}$ metacarpal as a variant in type I diabetes mellitus. \\
Ocronosis/Alkaptonuria. & Kesin beck disease. \\
\hline
\end{tabular}

\section{Management}

Patients with Pseudopseudohypoparathyroidism usually do not need specific treatment or further follow-up, but symptomatic treatment of related complication can be considered depending upon situations.

\section{Acknowledgement}

None.

\section{Conflict of interest}

Authors declare there is no conflict of interest in publishing the article.

\section{References}

1. AHO/PPHP case report.

2. Giovanna Mantovani. Endocrinology and Diabetology Unit, Department of Medical Sciences, Universita` degli Studi di Milano, Fondazione Istituto di Ricovero e Cura a Carattere Scientifico Ca' Granda Ospedale Maggiore Policlinico, Milan, Italy; 2012.

3. Esten W. Ray, Lytt I. Gardner, youngest case of psudo psudo hypoparathyrodism, Department of Pediatrics, State University of New York, Upstate Medical Center.

4. Davies SJ, Hughes HE. Imprinting in Albright's Hereditary Osteodistrophy. J Med Genet. 1993;30:101-3.
5. Albright F, Burnett CH, Smith CH, Parson W. Pseudohypoparathyroidism: "Seabright-Bantam sindrome. Endocrinology. 1942;30:922-932.

6. Lalande M. Imprints of disease at GNAS1. J Clin Invest. 2001;107:793-4.

7. Eyre WG. Reed WB. Albright hereditary osteodystrophy with cutaneous bone formation. Arch Dermatol. 1971;104:634-642.

8. Farfel Z, Friedman E. Mental deficiency in pseudohypoparathyroidism type I is associated with Ns-protein deficiency. Ann Intern Med. 1986;105(2):197-9.

9. Wilson LC. Albright's hereditary osteodystrophy. J Pediatr Endocrinol Metab. 2006;19(Suppl 2):671-673,105:197-199.

10. Long DN, McGuire S, Levine MA, et al. Body mass index differences in pseudohypoparathyroidismtype 1aversuspseudopseudohypoparathyroidism may implicate. J Clin Endocrinol Metab. 2011;96(10):3020-3030.

11. Albright F, Forbes AP, Henneman PH. Pseudopseudohypoparathyroidism. Trans Assoc Am Physicians. 1952;65:337-350.

12. Farfel $\mathrm{Z}$, Bourne HR, Iiri T. The expanding spectrum of $\mathrm{G}$ protein diseases. N Engl J Med. 1999;340:1012-20.

13. Weinstein LS, Shuhua Y, Warner DR, et al. Endocrine manifestations of stimulatory $\mathrm{G}$ protein a-subunit mutations and the role of genomic imprinting. Endoc Rev. 2001;22:675-705.

14. Marcel roche. Case of PPHP (pseudo-pseudohypoparathyrodism). Journal of clinical endocrinology and metabolism. 1955; 15(8):964-969. 